\title{
Über die Beziehung der Aktionsströme der Grosshirnrinde zu den Netzhautströmen des Menschen.
}

\author{
Von \\ Kōiti Motokawa und Tosisada Mita. \\ (本川弘一) (三田俊定) \\ (Aus dem Zweiten Physiologischen Institut der Tohoku \\ Kaiserlichen Universität, Sendai. Vorstand: \\ Prof. K. Motokawa.)
}

\section{Einleitung.}

Seit die alte Untersuchung Catons (1875) von Kornmüller ${ }^{1)}$ und Fis cher ${ }^{2}$ bestätigt wurde, hat die Potentialveränderung an der Grosshirnrinde bei Belichtung der Augen bei verschiedenen Tieren eingehende Untersuchungen erfahren. Bartley und B isho $\mathrm{p}^{3)}$ fanden, dass eine Reihe der Potentialschwankungen an der optischen Grosshirnrinde des Kaninchens auf elektrische Reizung des optischen Nerven hin auftreten. W an $\mathrm{g}^{4)}$ konnte mittels der konzentrischen Nadelelektroden nach Adrian und Bronk aus dem occipitalen Pol des Kaninchens je eine Potentialschwankung am Anfang und am Ende einer Belichtung der Augen ableiten und bezeichnete diese Schwankungen als "On"-Effekt resp. "Off"-Effekt. Bartle ${ }^{5)}$ stellte eine vergleichende Untersuchung der kortikalen Potentialschwankungen mit dem Elektroretinogramm des Kaninchens an und fand, dass die beiden Erscheinungen ein ganz gleiches Verhalten gegen die Art und Weise der optischen Reizung zeigen.

Jasper ${ }^{6)}$ und Cruikshank $k^{7}$ führten derartige Untersuchungen am Menschen aus und berichteten, dass sich dem "On"-Effekt bei Tieren entsprechende Potentialschwankungen auch bei der Ableitung von der Kopfoberfläche des Menschen nachweisen lassen. Bei den von diesen Autoren erhaltenen Elektrenkephalogrammen macht sich hauptsächlich ein elektropositiver Ausschlag auf dem Hinterkopf geltend, dem unter Umständen eine langsame elektronegative Schwankung folgt. Obgleich sie auch einige den erwähnten Schwankungen 
vorauseilende Oszillationen beschreiben, scheinen die letzteren von den spontanen Schwankungen des Hirnpotentials kaum unterscheidbar zu sein.

Mita ${ }^{8)}$ untersuchte die Beziehung zwischen der Latenzzeit der $a$-Wellen-Unterdrückung und der Reaktionszeit und machte uns darauf aufmerksam, dass die Belichtung nicht nur die $\alpha$-Wellen zum Verschwinden bringt, sondern auch typische Potentialschwankungen hervorruft. Sie bestehen aus zwei elektronegativen und -positiven Ausschlägen, an die sich eine langsame schwach elektronegative Schwankung unter Uinständen anschliesst. Der erste Ausschlag könnte bei einer schwachen Belichtung wenigstens scheinbar ausbleiben, aber der zweite elektropositive trat immer deutlich auf und stimmte zeitlich mit der Reaktionsbewegung des Fingers annährend iiberein. In vorliegender Untersuchurg bedienten wir uns dieser Eigenschaft, indem wir die Reaktionszeit immer neben dem Elektrenkephalogramm mit registrierten, um unseren „On"-Effekt (O.E.) leicht aufsuchen zu können. Denn der letztere ist bei nicht ausgeprägten Fällen schwer von der spontanen Schwankung des Hirnpotentials zu unterscheiden. Ein Beispiel des O. E. ist in Abb. 1. veranschaulicht.

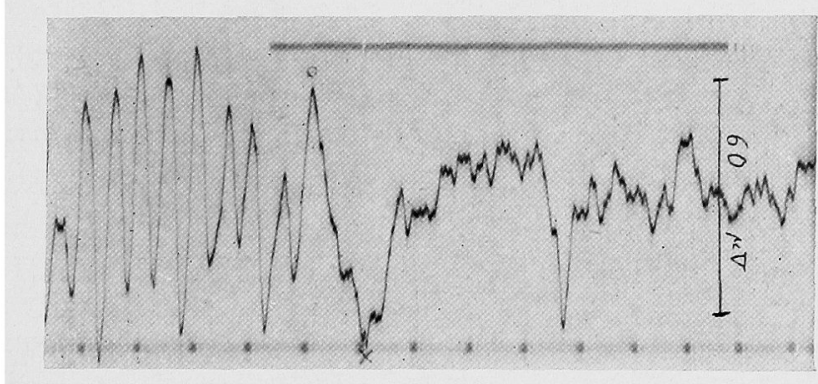

Abb. 1. Der „On“-Effekt auf Belichtung der Augen bei der Stirn-Hinterhaupt-Ableitung. Das Linienstück oberhalb der Kurve zeigt die Belichtungsdauer. Das Moment der Fingerreaktion auf Belichtung ist auf diesem Linienstück mit einer Unterbrechung markiert. $O$ : In bezug auf den am Hinterhaupt elektronegativen Ausschlag. $\quad \times$ : Elektropositiver Ausschlag. Zeit in 1/5 Sek.

Unser O. E. stimmt im zeitlichen Verhältnis mit demselben von Jasper und Cruikshank im grossen und ganzen überein, aber die beiden sind mit dem „On"-Effekt bei Tieren nicht ganz in Übereinstimmung; z. B. bei Tieren lässt sich der „Off"-Effekt immer ebenso deutlich wie der "On"-Effekt nachweisen (Bartley und Wang), 
während sich die dem ,Off"-Effekt entsprechende Schwankung beim Menschen kaum bemerkbar macht.

Rohracher ${ }^{9)}$ beobachtete auch derartige Potentialschwankungen beim Menschen, führte sie aber auf das Kunstprodukt infolge der körperlichen Bewegung der Versuchsperson zurück. Es muss also genau untersucht werden, ob derartige Schwankungen wirklich auf körperlichen Bewegungen beruhen oder nicht. Jasper und Cruikshank schreiben diese Schwankungen ohne weiteres der Grosshirnrinde zu, aber bei der Ableitung von der Kopfoberfläche können Potentialschwankungen verschiedener Herkunft in Betracht kommen. In vorliegender Arbeit wurden solche Fehlerquellen ausgeschlossen und das Wesen des sogenannten O. E. beim Menschen einer eingehenden Kritik unterworfen.

\section{Methodik.}

Die Methodik der Elektrenkephalographie ist dieselbe wie bei der Arbeit von Mita. ${ }^{8)}$ Ein Unterschied besteht in der Anwendung von Zinnfolienelektroden zur Ableitung. Die Berührung der Elektroden mit der Haut wurde durch eine den Strom gut leitende Kochsalzsalbe gesichert, die Dr. K. Matuda für uns hergestellt hatte. Diese Salbe wird von ihm bei der Elektrokardiographie mit Erfolg angewandt und bewährt sich sehr gut, um die Polarisation der Haut herabzusetzen. Wir sind ihm für seine Freundlichkeit zu herzlichem Dank verpflichtet. Die Folienelektroden sind auch für Untersuchungen der Augenbewegungen sehr geeignet, wie unten gezeigt wird.

Die Registrierung der Potentialschwankungen geschah mittels des Schleifenoszillographen von Yokogaw (Schleife vom H-Typus) und eines Vierstufenverstärkers, aber wenn es auf den langsamen Verlauf der Schwankungen ankam, wurden auch ein dreistufiger Gleichstromverstärker und die Oszillographenschleife vom D-Typus (Yokogawa) zur Anwendung gebracht.

\section{Versuchsergebnisse.}

1. Sind reflektorische Lidbewegungen für den "On" nicht verantwortlich?

Es ist bekannt, dass Augen- bzw. Lidbewegungen auf das Elektrenkephalogramm einen merklichen Einfluss ausüben. Ein Beispiel ist in Abb. 2 illustriert, woraus man entnehmen kann, dass die durch einen willkürlichen Lidschlag verursachte grosse Schwankung dem 
O.E. etwas ähnlich ist. Es fragt sich nun, ob unser O. E. nicht durch reflektorische Lidbewegungen bei der Augenbelichtung verursacht wird. Diese Frage scheint etwas berechtigt, weil das zeitliche Verhältnis des $\mathrm{O}$. E. als ein reflektorisches Phänomen nicht ganz unbegreiflich ist; der erste elektronegative Ausschlag tritt augenscheinlich mit einer Latenz von gleicher Grössenordnung wie die Reflexzeit auf, und der elektropositive fällt zeitlich mit der Reaktionszeit

Abb. 2. Die durch einen willkürlichen Lidschlag hervorgerufene Potentialschwankung bei der Stirn.Hinterhaupt-Ableitung. Zeit in $1 / 5$ Sek. des Fingers zusammen, wie eingangs erwähnt.

Abgesehen von diesem Umstand, ist der Effekt der Lidbewegung bei der gewöhnlichen Elektrenkephalographie, d. h. bei der Hinterhaupt-Stirn- oder Hinterhaupt-Ohrlappen-Ableitung so wirksam, dass eine geringste kaum fühlbare Lidbewegung eine grosse Potentialschwankung im Elektrenkephalogramm hervorrufen kann. Wegen dieses Umstandes ist der O. E. insofern nicht ganz einwandfrei, als der Nachweis nicht erbracht worden ist, dass er auch beim strengsten Ausschluss der Lidbewegung noch auftritt. Um diesen Beweis zu erbringen, ist eine Methode unbedingt notwendig, mit der man auch die kleinste Lidbewegung mit Sicherheit nachzuweisen vermag.

Die Methode, welche wir nach vierfachen Vorprüfungen gewählt haben, ist folgende: Das freie Ende einer auf den Kopf mit Isoliermaterial befestigten Zinnfolienelektrode wurde am beweglichsten Teil des oberen Lides mit der erwähnten Pasta leicht angeklebt, während die andere indifferente Zinnfolienelektrode auf die Stirn aufgesetzt wurde. Bei dieser Anordnung der Elektroden wurde die differente Elektrode bei jeder Bewegung des Lides in Mitbewegung gesetzt, die bei hoher Empfindlichkeit des Verstärker- OszillographSystems eine grosse Potentialschwankung hervorrief. Auf diese Weise konnten wir jede Spur der Lidbewegung mit Sicherheit nachweisen.

Falls die Versuchsperson geübt genug war, liess sich keine Lidbewegung trotz dieser hohen Empfindlichkeit nachweisen, vorausgesetzt dass die Belichtung nicht blendend war. War die Versuchsperson dagegen ungeübt, oder die Belichtung zu stark, so fanden 
Lidbewegungen natiirlich statt, und dementsprechend liessen sich Potentialschwankungen mehr oder weniger deutlich je nach dem Grad der Lidbewegungen bemerken. Bei solchem Fall fühlten achtsame Versuchspersonen immer eine subjektive Bewegungsempfindung, wie schwach die Bewegung auch sein mochte, und umgekehrt empfanden sie keine Bewegung, wenn Potentialschwankungen ausblieben. Diese Tatsache weist einerseits auf die Zuverlässigkeit unserer Methode und andererseits darauf hin, dass eine Lidbewegung bei der Belichtung der Augen nicht immer auftritt. Angesichts der Tatsache, dass der O. E. bei der Belichtung immer auftritt, wenn die Lichtstärke nicht unter der Schwelle bleibt, dürften Lidbewegungen nicht mehr als eine Ursache des $O$. E. betrachtet werden.

\section{Ist der "On"-Effekt von Augenbeivegungen abhängig?}

Die Bewegungen des Körpers, insbesondere der Augen können bei der Elektrenkephalographie zu merklichen Potentialschwankungen führen, so dass derartige Versuche bei der körperlichen Ruhe und mit fixierten Augen ausgeführt werden müssen.

Fuir die so verursachten Potentialschwankungen ist die Verschiebung der Elektrode in meisten Fällen verantwortlich, aber unter Umständen können die Aktionsströme der an der Bewegung beteiligten Muskeln in Betracht kommen. Was unseren „On"-Effekt anbetrifft, so kommen diese Faktoren kaum in Frage. Denn die Elektroden wurden bei unserer Untersuchung mit Heftpflastern auf den Kopf fest fixiert, um etwaiger Verschiebung vorzubeugen, und doch trat der O. E. immer deutlich auf. Die in Frage kommenden Aktionsströme der Muskeln sind nicht schwer auszuschliessen, weil ihr Verlauf von demjenigen des $O$. E. ganz verschieden sein soll.

Als eine noch wahrscheinlichere Ursache ist die Bewegung eines in sich eine Potentialdifferenz tragenden Organs wie z. B. die des Auges anzuführen. Dabei erfährt die Potentialverteilung um das betreffende Organ eine Veränderung, die sich als Potentialschwankungen im Ableitungskreis bei der Elektrenkephalographie bemerkbar machen kann. Wir sind imstande, umgekehrt aus derartigen Potentialschwankungen die Bewegung des betreffenden Organs kennenzulernen.

Schott ${ }^{10)}$ und Myersis unternahmen auf Grund dieses Prinzips Augenbewegungen elektrisch zu registrieren. Mowrer, Ruch und Miller ${ }^{12)}$ haben den Beweis erbracht, dass die die Augenbewegung 
begleitende Potentialveränderung tatsächlich auf dem Bestandpotential der Netzhaut beruht.

Nun erhebt sich die Frage, ob unser O. E. nicht in dieser Weise durch reflektorische Augenbewegungen bei der Belichturig verursacht wird. Findet irgend eine Augenbewegung bei der Belichtung statt, so muss die so entstandene Potentialveränderung wenigstens bei der gewöhnlichen Ableitung mehr oder weniger das Elektrenkephalogramm beeinflussen. Also versuchten wir in erster Linie zu entscheiden, ob eine Augenbewegung tatsächlich bei der Belichtung mit den bei der Elektrenkephalographie von uns angewandten Reizlichtern immer stattfindet oder nicht, und zweitens zu untersuchen, wie willkürliche Augenbewegungen verschiedener Grade das Elektrenkephalogramm beeinflussen können.

Zum Nachweis der Augenbewegungen wurde die oben erwähnte elektrische Methode angewandt, nachdem ihre Brauchbarkeit zu diesem $Z$ weck bestätigt worden war. Die Zinnfolienelektroden wurden auf symmetrische Stellen der Gesichtshaut, z. B. bei horizontalen Augenbewegungen beiderseits der äusseren Augenwinkel und bei vertikalen auf die Supra- und Infraorbitalgegend aufgesetzt. Die Versuchsperson folgt einem hin und her pendelnden Merkmal eines Metronoms mit den Augen, so dass die Potentialschwankungen mit dementsprechender Periode und Amplitude registriert wurden. Das Ausmass der Augenbewegungen wurde bei geeigneter Wahl der Periode mit der Amplitude des Merkmals proportional verändert, so dass eine quantitative Beziehung zwischen dem Bewegungswinkel der Augen und der Amplitude der Potentialschwankungen leicht ermittelt wurde.

Ein Beispiel derartiger Potentialschwankungen ist in Abb. 3 veranschaulicht. Wie Fenn und $\mathrm{Hursh}{ }^{13)}$ schon hinwiesen, hängt die Amplitude der Potentialschwankungen mit dem Bewegungswin-

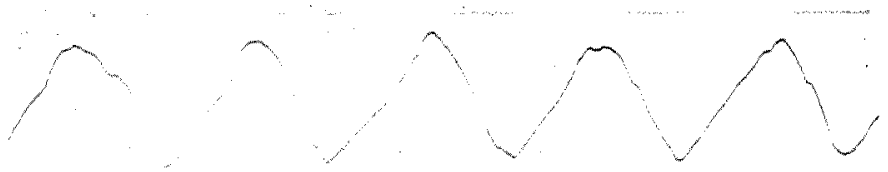

Abb. 3. Die nach der elektrischen Methode registrierten horizontalen rhythmischen Augenbewegungen.

Ableitelektroden beiderseits an den äusseren Augenwinkeln aufgesetzt. Bewegungswinkel 24,5 . Die Linienstücke oberhalb der Kurve zeigen die Periode der Augenbewegungen. Zeit in 1/5 Sek. 
kel der Augen quantitativ zusammen; theoretisch ist zu erwarten, dass die Amplitude $A$ mit dem Produkt aus dem Bestandpotential der Netzhaut E und dem Sinus der Hälfte des Bewegungswinkels der Augen $\theta$ proportional ist, d. h. : $A=k E \sin \theta / 2$, wobei $k$ den Proportionalitätsfaktor bedeutet und von der Ableitungsbedingung, individueller Verschiedenheit usw. abhängig ist, und aus unserer Untersuchung stellte sich heraus, dass diese Formel für vertikale sowie horizontale Bewegungen der Augen annähernd gültigi ist, wie aus Tabelle I und II ersichtlich wird.

\section{Tabelle I.}

Potentialschwankungen bei horizontalen Augenbewegungen.

\begin{tabular}{c|c|c}
\hline \multirow{2}{*}{$\begin{array}{c}\text { Bewegungs- } \\
\text { windel in } \\
\text { Graden }\end{array}$} & \multicolumn{2}{|c}{$\begin{array}{c}\text { Amplituden der Poten- } \\
\text { tialschwankungen } \\
\text { in mm. }\end{array}$} \\
\cline { 2 - 3 } & Beobachtet & Berechnet \\
7,4 & 35 & 34,4 \\
3,7 & 20 & $17, ?$ \\
2,8 & 11 & 13,1 \\
1,1 & 5 & 5,1 \\
12,5 & 62 & 61,5 \\
6,45 & 31 & 31,8 \\
3,29 & 18 & 15,9 \\
1,61 & 7 & 8
\end{tabular}

Tabelle II.

Potentialschwankungen bei vertikalen Augenbewegungen.

\begin{tabular}{c|c|c}
\hline $\begin{array}{c}\text { Bewegungs- } \\
\text { winkel in } \\
\text { Graden }\end{array}$ & $\begin{array}{r}\text { Ampituden der Poten- } \\
\text { tialschwankungen } \\
\text { in mm. }\end{array}$ \\
\hline 16,1 & Beobachtet & Berechnet \\
8,3 & 74 & 85,4 \\
4,1 & 40 & 44 \\
2,1 & 23 & 21,8 \\
13 & 11,2
\end{tabular}

Trotz dieser empfindlichen und ziemlich exakten Methode liess sich keine reflektorische Augenbewegung in der Mehrzahl der Fälle nachweisen, wenn die $\mathrm{Au}$ gen der Versuchsperson auf einen roten schwach leuchtenden Punkt fixiert gehalten wurden. Lag die Versuchsperson im Gegensatz dazu ohne Fixierpunkt im Dunkeln, wie bei der gewöhnlichen Elektrenkephalographie, so konnte die Gesichtslinie unter Umständen unruhig wandern und auf Belichtung hin reflektorisch nach der Leuchtfläche gerichtet werden. Dabei trat natürlich eine deutliche Potentialschwankung auf. Aber diese Tatsache deutet nicht ohne weiteres darauf hin, dass derartige Augenbewegungen bei der gewöhnlichen Elektrenkephalographie ebenso grosse Potentialschwankungen hervorrufen, denn eine Potentialschwankung, die sich bei einer Ableitungsweise deutlich bemerkbar macht, kann sich bei einer anderen gar nicht nachweisen lassen.

In Wirklichkeit ist die Empfindlichkeit für die Augenbewegung bei der Hinterhaupt-Stirn-Ableitung der in bezug auf das Auge symmetrischen Ableitung gegenüber stark reduziert; eine solche Augen- 
bewegung, wie eine mittelmässige Potentialschwankung bei der letzteren Ableitung verursachen würde, kann bei der ersteren gar keme Potentialschwankung hervorrufen (Abb. 4 b), während eine grosse Augenbewegung wie z. B. von $16^{\circ}$ natürlich wirksam ist (Abb. 4 a). Es ist in dieser Beziehung hervorzuheben, dass die Empfindlichkeit bei der Hinterhaupt-Scheitel-Ableitung noch schlechter als bei der Hinterhaupt-Stirn-Ableitung ist; selbst eine so grosse Bewegung wie von $16^{\circ}$ konnte keine bemerkbare Potentialschwankung verursachen (Abb. $4 \mathrm{c})$.

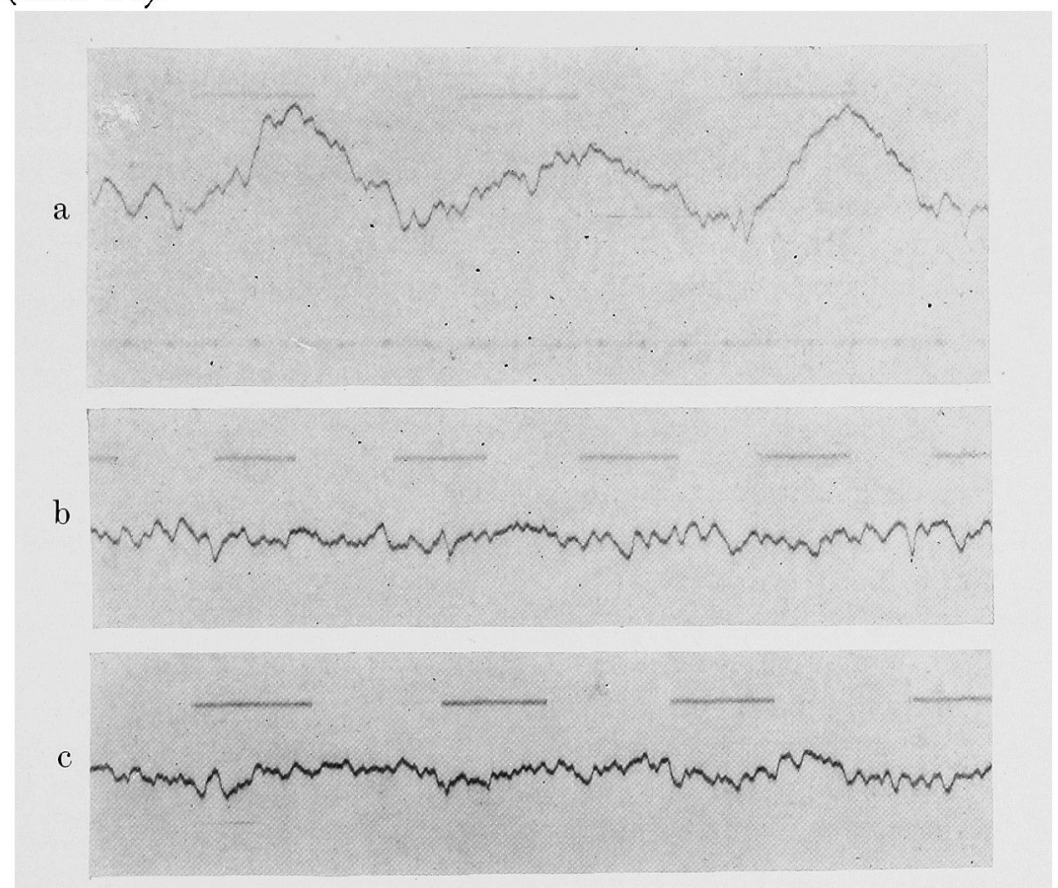

Abb. 4. Die durch Augenbewegungen hervorgerufenen Potentialschwankungen bei verschiedenen Ableitungen.

a : Ableitung Stirn-Hinterhaupt. Augenbewegungen von $16^{\circ}$, vertikal. b: Dieselbe Ableitung. Bewegungen von 2,3 $3^{\circ}$ vertikal. c: Ableitung Scheitel-Hinterhaupt. Bewagungen von $16^{\circ}$, vertikal. Kleinere Wellen sind Hirnstöme.

Aus dieser Untersuchung geht hervor, dass die Potentialschwankung vom okularen Ursprung nur dann deutlich zum Ausdruck kommt, wenn wenigstens eine Elektrode in der Nähe der Augen liegt. Da eine Elektrode bei der gewöhnlichen Elektrenkephalographie auf der $^{2}$ Stirn oder auf den Ohrlappen, also verhältnismässig nahe den Augen 
liegt, sind wir noch nicht sicher, dass die Augenbewegung als das ursächliche Moment des „On"-Effektes nicht in Frage kommt. Um zu sehen, ob der O.E. durch willkürliche Ablenkung der Gesichtslinien tatsächlich eine wesentliche Veränderung erfährt oder nicht, wurde folgende Untersuchung angestellt.

Ein roter Fixierpunkt wurde in verschiedenen Richtungen und Abständen vom darzubietenden Reizlicht angebracht. Die Augen der Versuchsperson wurden auf diesen Punkt fixiert gehalten und auf die Belichtung hin so schnell wie möglich von diesem Punkt nach der Leuchtfläche gerichtet, aber der übliche „On"-Effekt erfuhr durch diese Bewegung keine wesentliche Veränderung, sei es nun Hebung, Senkung, Konvergenz oder Divergenz der Gesichtslinien, wem die Bewegung etwa $10^{\circ}$ nicht überstieg. Keine so ausgiebige Wanderung der Gesichtslinien findet bei geübten Versuchspersonen selbst im Dunkeln statt, so dass das konstante Auftreten des O. E. von diesem Standpunkte aus nicht mehr erklärt werden kann.

\section{3. Über das Verhalten des ,On"-Effektes bei Belichtung der geschlossenen Augen.}

Wie oben erwähnt, kamen Lid- und Augenbewegungen als ursächliche Momente für den "On"-Effekt in erster" Linie in Betracht. Dieser Umstand veranlasste uns, einige Untersuchungen mit geschlossenen Augen, aber unter der sonst gleichen Versuchsbedingung anzustellen, denn die Lid- und Augenbewegungen bei diesem Zustand der Augen sollten aufs Minimum beschränkt werden. Die Ergebnisse dieser Untersuchung widersprachen scheinbar dem oben gezogenen Schluss, dass die Lid- und Augenbewegungen nicht für den O. E. verantwortlich sind, denn bei geschlossenen Augen trat der letztere nicht mehr auf (Abb. 5 b) oder wenn er auch vereinzelt aufzutreten schien, war das Ergebnis recht zweideutig Abb. 5 a).

Sollte dieses Ausbleiben des O. E. auf der schwachen Intensität des durch das Lid hindurch gehenden Reizlichtes beruhen, so würde der Widerspruch fortfallen, aber der Sachverhalt war nicht so einfach ; bei offenen Augen lässt sich der $\mathrm{O}$. E. bei einer höchst schwachen, d. h. eben schwelligen Belichtung mit Sicherheit nachweisen, während bei geschlossenen Augen mit einem genügend starken Licht, z. B. mit einer elektrischen Lampe von 200 Watt kein positives Resultat zu erzielen ist, und zwar empfindet die Versuchsperson dabei sogar eine grössere Helligkeit als beim Versuch mit offenen Augen. 


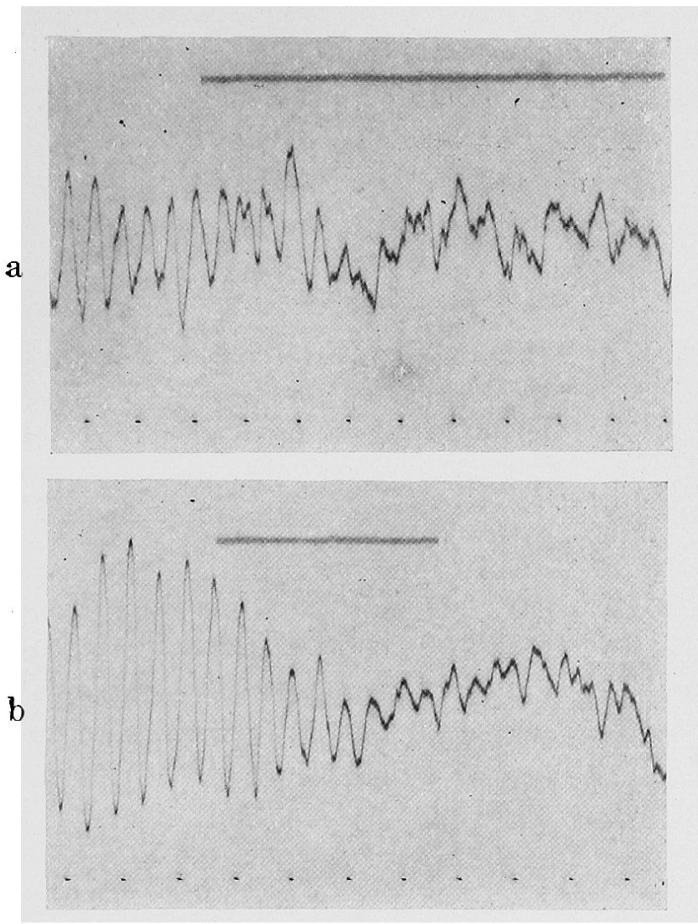

Abb. 5. Das Verhalten des „0n“-Effektes bei Belichtung der geschlossenen Augen.

$a$-Wellen werden durch Belichtung unterdrückt. a : "On"-Effekt schwach. b: Er bleibt vollständig aus. Zeit in $1 / 5$ Sek.
Beim letzteren Fall geht das Versch winden der $\alpha$-Wellen mit dem Einsetzen des O.E. einher. Im Gegensatz dazu beim Versuch mit geschlossenen Augen gehen die $\alpha$-Wellen zwar auf Belichtung hin verloren, und doch macht sich kein O.E. bemerkbar.

Nach Adrian und Matthew $\mathrm{s}^{14)}$ ist ein diffuses Licht zur Unterdrückung der $\alpha$ Wellen weniger wirksam als ein optische Bilder produzierendes Licht. Es fragt sich nun, ob ein diffuses Licht wie bei geschlossenen Augen noch weniger wirksam sei, um den O. E. hervorzurufen. Um diese

Frage zu beantworten, wurden diffuse Lichter verschiedener Stärke hergestellt, und damit wurden die offenen Augen belichtet. Das Ergebnis war recht eindeutig; der O. E. kam trotz der diffusen Eigenschaft des Reizlichtes ebenso deutlich wie bei einem nicht diffusen Reizlicht zustande.

\section{Dor Einfluss der Lichtfarbe auf den „On"-Effekt.}

Ausser den oben genannten Bedingungen musste noch eine andere, nämlich der Unterschied der Lichtfarbe in Erwägung gezogen werden. Das durch das Lid hindurch gehende Licht ist gewöhlich gelblich rot gefärbt, wenn auch ein weisses Licht von aussen her angewandt wird. Es wurden verschieden gefärbte Lichter mittels Glasfilter von bekannter selektiver Durchlässigkeit hergestellt und ihre 
Helligkeit durch die Flimmerphotometrie bestimmt. Die Lichtquelle war dieselbe für alle Filter, nämlich die 100 Watt-Lampe von M azda. Mit den so hergestellten Lichtern wurden die offen und in einem schwach helladaptierten Zustand gehaltenen Augen in einem Abstand von etwa $100 \mathrm{~cm}$ belichtet. Die Resultate sind in Abb. 6 wiedergegeben.

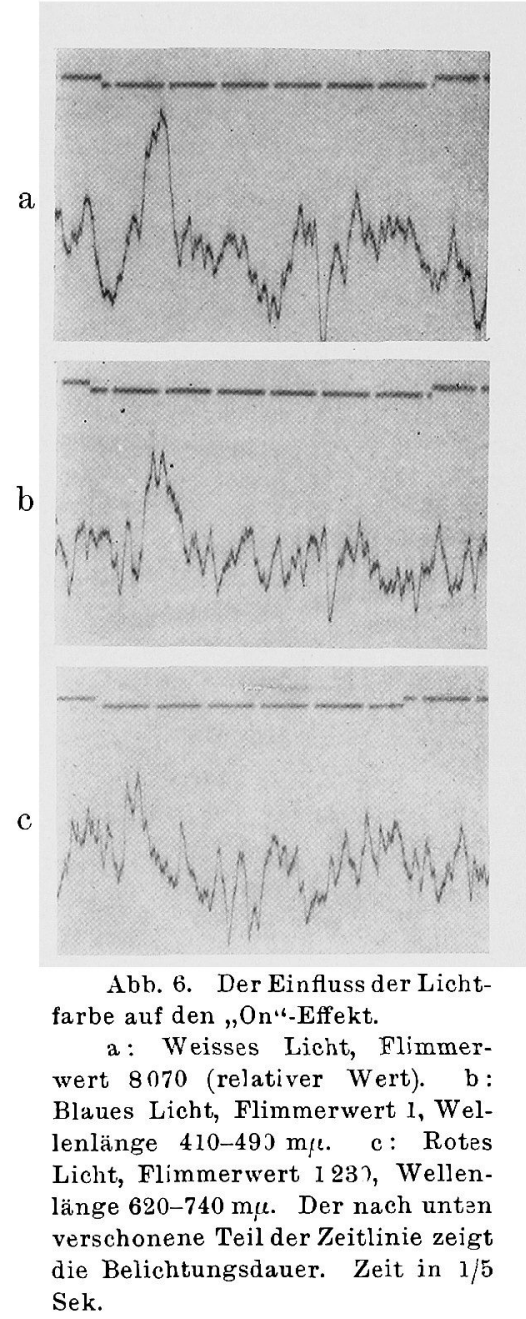

Obgleich die angewandten Lichter dem Flimmerwert nach in Verhältnissen wie Weiss : Rot : Blau $=8070: 1230: 1$ standen, ordneten sie sich der Wirksamkeit nach in der Reihe Weiss > Blau $>$ Rot; merkwürdig ist besonders, dass die Helligkeit des roten Lichtes etwa 1000 fach so gross wie diejenige des blauen Lichtes ist, und doch dass die Wirksamkeit des. ersteren viel kleiner als die des letzteren ist. In Anbetracht der Tatsache, dass das Reizlicht bei geschlossenen Augen rötlich gefärbt ist, wird die vorliegende Untersuchung ausreichen, um den Unterschied zwischen den Ergebnissen bei offenen und geschlossenen Augen verständlich zu machen.

Nach Himstedt und $\mathrm{Na-}$ gel $1,{ }^{15)} \mathrm{Piper},{ }^{16)} \mathrm{Bross}$ a und Kohlrausch, ${ }^{17)}$ Fröhlich ${ }^{18)}$ und anderen ist der Belichtungsstrom des Kaltblüterauges in gleicher Weise von der Wellenlänge des Reizlichtes vielfach abhängig, und nach Sachs ${ }^{19}$; ist auch beim Menschen das rote Licht in bezug auf das Elektroretinogramm weniger wirksam als das weisse oder das blaue Licht. In Anbetracht dieser Tatsachen liegt der Gedanke nahe, dass unser „On"-Effekt auch von retinalem Ursprung sein kann. 
Wie oben erwähnt, kann sich das Bestandpotential der Netzhaut bei verhältnismässig grossen Augenbewegungen für die Elektrenkephalographie geltend machen, und somit liegt die Wahrscheinlichkeit recht nahe, dass das Belichtungspotential, welches einige zehntel $\mathrm{mV}$ betragen kann, bei der Elektrenkephalographie mit registriert wird. Besitzt das Belichtungspotential wirklich mit dem O. E. irgend einen Zusammenhang, so muss der letztere von der Ableitungsbedingung vielfach abhängig sein. Wir haben nun durch systematische Untersuchungen in dieses Verhältnis Klarheit zu bringen.

\section{Die Abhängigkeit des „On“-Effektes von der Ableitungsweise.}

Es wurde in erster Linie untersucht, wie sich der O. E. verhält, wenn eine Elektrode auf dem Hinterhaupt fixiert gehalten und die andere von der Stirn nach dem Hinterhaupt hin verschoben wird. Das Ergebnis ist in Abb. 7 wiedergegeben. Wenn die verschiebbare Elektrode von der Stirn nach der Schläfe hin verschoben wird, so reduziert sich die Amplitude des O. E. etwas ohne dass sein zeitlicher Verlauf eine wesentliche Änderung erfährt (Abb. a und b). Wird der Abstand zwischen den beiden Elektroden noch verkleinert,

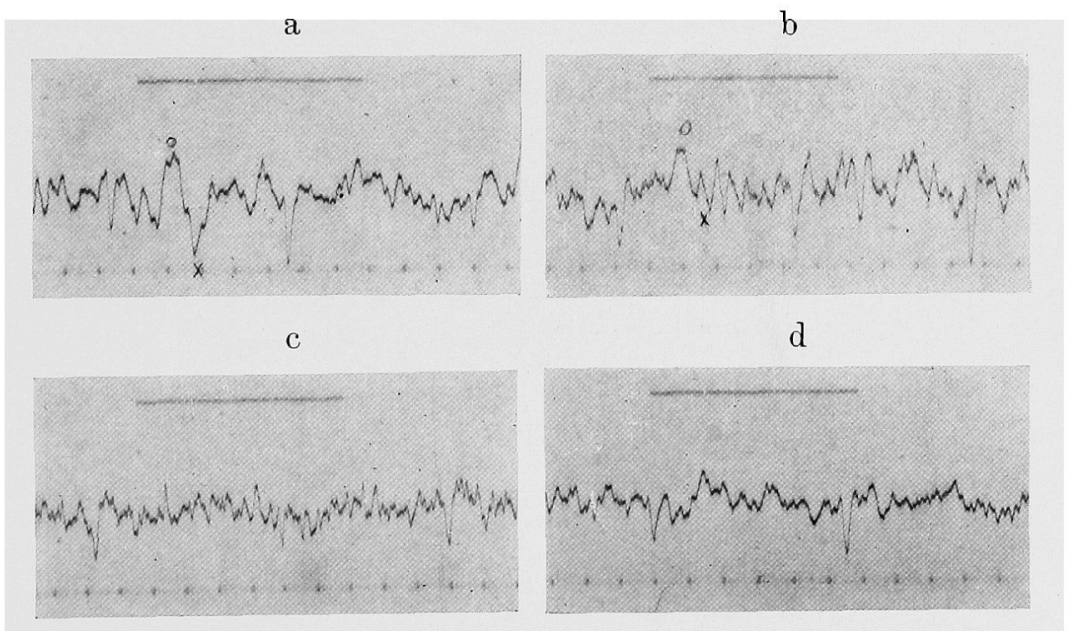

Abb. 7. Die Abhängigkeit des „On“-Effektes von der Ableitungsweise.

Untersucht in einem Dunkelzimmer mit einem roten, schwach leuchtenden Fixierpunkt. Ableitung. a: Hinterhaupt-Stirn. b: Hinterhaupt-Schläfe. c: Hinterhaupt-3 cm oberhalb des Proc. mastoid. d: Hinterhaupt-Scheitel. Zeit in $1 / 5$ Sek. 
indem die vordere Elektrode auf eine Hautstelle hinter dem Ohr aufgesetzt wird, so kommt kein dem O. E. entsprechender Ausschlag in der Mehrzahl der Fälle zustande (Abb. $7 \mathrm{c}$ ). Das gleiche gilt auch für die Scheitel-Hinterhaupt-Ableitung (dieselbe Abb. d).

Kurz zusammengefasst lässt sich sagen: die Amplitude des O. E. wird desto stärker reduziert, je entfernter die differente Elektrode von den Augen liegt. Es ist hervorzuheben, dass der O. E. nur schwach erscheint; wenn die beiden Elektroden auf der hinteren Hälfte des Kopfes liegen. Diese Tatsache weist darauf hin, dass der O. E. von der vorderen Hälfte des Kopfes, aber nicht von der optischen, Hirnrinde herrührt, wie vermeint.

Bei einer anderen Reihe der Versuche wurde eine Elektrode auf die Nasenwurzel fixiert gehalten, um sie möglichst nahe den vorderen Polen der beiden Augen zu halten, und die andere von dem Hinterhaupt nach vorn hin verschoben. Das Resultat ist in Abb. 8 veranschaulicht. Die daraus ohne weiteres hervorgeht, bleibt der O. E.

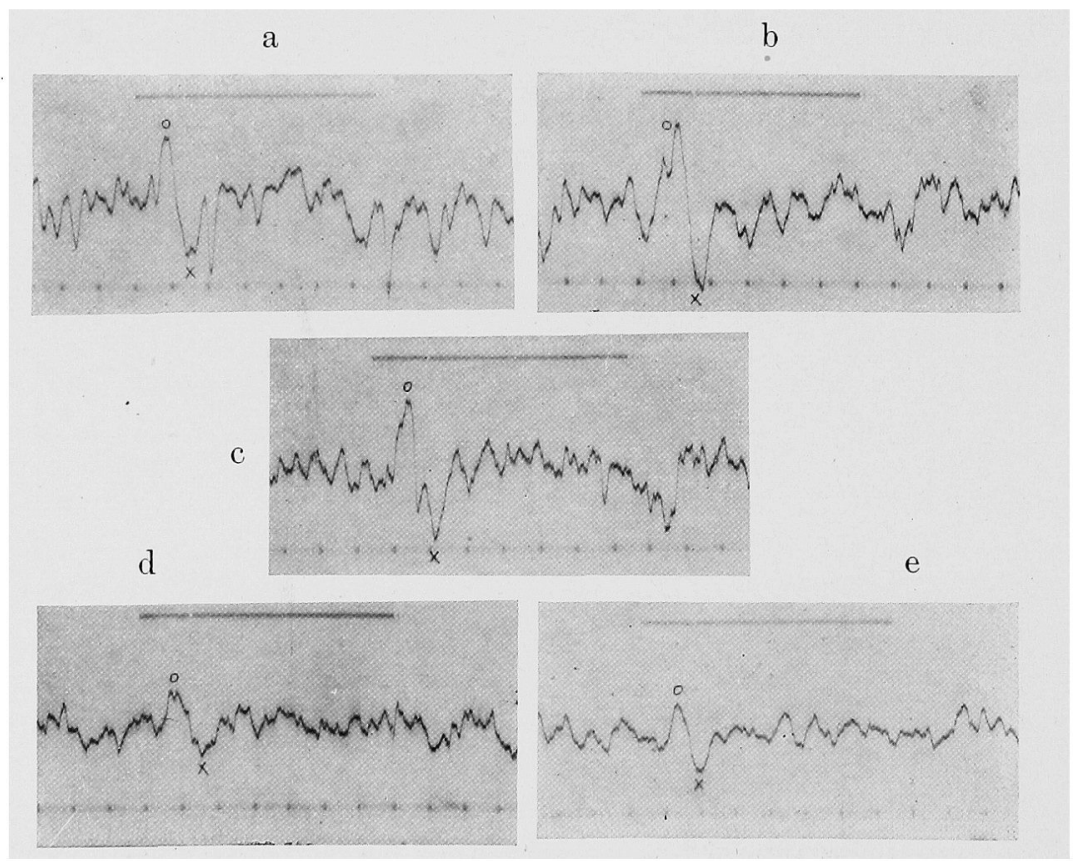

Abb. 8. Die Abhängigkeit des „On“-Effektes von der Ableitungsweise.

Ableitung. a: Nasenwurzel-Hinterhaupt. b: Nasenwurzel-Scheitel.

c: Nasenwurzel $-3 \mathrm{~cm}$ oberhalb des Proc. mastoid. d : Nasenwurzel-Schläfe.

e: Nasenwurzel-Stirn. Zeit in $1 / 5$ Sek. 
gross genug, solange die verschiebbare Elektrode hinter den Augen liegt ( $a, b$, und $c$ ), aber ihre Grösse wird stark reduziert, wenn die verschiebbare Elektrode über den hinteren Pol der Augen hinüber der vorderen fixierten Elektrode genähert wird (d und e). Dies Verhalten ist unter der Voraussetzung erst begreiflich, dass die Quelle dieser Potentialänderung an den Augen vorliegt.

\section{Die Identifizierung des ,On"-Effektes mit dem Elektroretinogramm.}

Um den O. E. mit dem Elektroretinogramm identifizieren zu diurfen, reicht die Feststellung der Lokalisation der EMK nicht ganz aus; es muss weiter festgestellt werden, dass die Form und der zeitliche Verlauf des O. E. mit denjenigen des Elektroretinogramms des Menschen völlig übereinstimmen.

Während Versuche über die Belichtungsströme der Netzhaut der Tiere reichlich vorliegen, sind solche beim Menschen noch recht spärlich. Sachs, ${ }^{19)} \mathrm{Hartline}^{20)}$ und Gröppel, Haass und Kohlrau$\mathrm{sch}^{21)}$ leiteten den Belichtungsstrom des Menschen mit grosser Mühe von der Kornea und der Schläfe ab, und konstatierten, dass das Elektroretinogramm des Menschen an Form und Verlauf mit demjenigen der Säugetiere beinahe übereinstimmt; die grösste Schwankung, welche als Eintritts-oder b-Schwankung bezeichnet wird, tritt zuerst auf, aber ein kleiner, umgekehrt gerichteter Ausschlag kann unter Umständen der ersteren vorausgehen (a-Schwankung). Der Eintrittsschwankung folgt gewöhnlich eine sekundäre Erhebung oder cSchwankung.

Der erste Ausschlag des O. E. tritt in derselben Richtung wie die Eintrittsschwankung des Elektroretinogramms auf, und der zweite stimmt mit dem absteigenden Schenkel der Eintrittsschwankung uberein, aber es gehört nur zu Ausnahmen, dass der absteigende Schenkel des Elektroretinogramms weit unter die Nullinie reicht, wie es beim zweiten Ausschlag des O. E. der Fall ist. Dies ist der einzige Unterschied zwischen dem O. E. und dem Elektroretinogramm.

Berïcksichtigt man aber, dass der O. E. bei fast allen Untersuchungen mittels eines Verstärkers von der Kapazität-WiderstandKopplung registriert wurde, so kann vom naturgetreuen Verlauf solch einer langsam ablaufenden Erscheinung natürlich keine Rede sein. Nur ein Gleichstromverstärker mit einer genügenden Empfindlichkeit gestattet uns den naturgetreuen Verlauf kennenzulernen. Wir kom- 
binierten einen Dreistufengleichstromverstärker mit der Yokogaw a schen Oszillographenschleife vom D-Typus (Empfindlichkeit 1,5 $\times$ $10^{-6} \mathrm{~V} / \mathrm{mm}$, Periode 1/150 Sek., Widerstand 8,5 Ohm) und konnten dieselbe Empfindlichkeit bei der Anwendung eines Vierstufenverstärkers und der Oszillographenschleife vom H-Typus (Empfindlichkeit $7,5 \times 10^{-5} \mathrm{~V} / \mathrm{mm}$, Periode $1 / 1000$ Sek., Widerstand 2 Ohm) erzielen.

Ein Beispiel der so registrierten Kurven ist in Abb. 9 illustriert. Wie daraus ohne weiteres hervorgeht, stimmt der Verlauf des O. E. mit demjenigen des Elektroretinogramms völlig überein, abgesehen davon, dass er von kleinen Schwankungen der Hirnströme überlagert ist. Aus diesen Untersuchungen dürften wir mit Recht schliessen, dass unser O. E. nichts anderes als der Belichtungsstrom der Netzhaut ist, und dass die Potentialschwankung an den Augen bei der Elektrenkephalographie unter Umständen in den Messungskreis hineinstreuen und somit eine grosse Konfusion hervorrufen kann.

Abb. 9. Der naturgetrene Verlauf des „On“-Effektes (mit einem Gleichstromverstärker und einem Oszillographen registriert).

Anfang und Ende der Belicntung mit Pfeilen angezeigt. Zeit in $1 / 5$ Sek.

\section{7. Über den Entstehungsort der elektrisehen Antwort auf rhythnische Augenbelichtung.}

Adrian und Matthews $\left.{ }^{14}\right)$ haben mitgeteilt, dass auf rhythmische optische Reize (,Flicker") periodische Potentialschwankungen auftreten. Jasper ${ }^{6)}$ hat den Flickereffekt nicht nur beim Menschen sondern auch bei Tieren bestätigt. Die Verschmelzungsfrequenz war 
dabei viel höher als beim Adrianschen Versuche, nämlich 40-50 pro Sek. und stimmte mit derjenigen der subjektiven Empfindung beinahe überein. Im Gegensatz dazu erwähnen Loomis, Harvey und Hobart, ${ }^{22}$ dass drei ihrer Versuchspersonen innerhalb einer Flickerfrequenz von 10-20 pro Sek. den Befund Adrians zeigten, während eine weitere Versuchspelson nur eine sehr geringgradige Wirkung eines Flickers erkennen liess. Rohracher ${ }^{23)}$ konnte bei seinem Versuch dieses Phänomen nicht beobachten, während Kornmüller ${ }^{24}$ den Adrianschen Befund teilweise bestätigen konnte. Bei den angeführten Arbeiten setzten einige Forscher (z. B. Adrian) wenigstens eine Elektrode in der Nähe der Augen, z. B. auf die Stirn auf, während bei anderen Fällen beide Elektroden verhältnismässig davon entfernt aufgesetzt wurden (bipolare Ableitung).

Da es keinem Zweifel unterliegt, dass sich der Belichtungsstrom der Netzhaut um die Augen verbreitet, lässt sich die Möglichkeit nicht von vornherein ausschliessen, dass die Flickerantwort, welche die bisherigen Forscher ohne weiteres auf das Hirn zurückgeführt haben, nicht von der Netzhaut hätte herrühren können. Bei unseren Versuchen konnten wir auch individuelle Verschiedenheiten bemerken, wie beim Versuche von Loomis und anderen; es liess sich keine fassbares Flickerantwort bei einer Versuchsperson, die sehr regelmässige $\alpha$-Wellen zeigte, beobachten, während sie bei einer anderen mit weniger entwickelten $\alpha$-Wellen recht ausgeprägt auftrat.

In einer Reihe der Versuche wurde die Nasenwurzel-SchläfeAbleitung angewandt, um die Flickerantwort an der Netzhaut am deutlichsten hervortreten zu lassen, wenn eine solche iberhaupt vorhanden ist, und in einer anderen Reihe wurden die Elektroden auf die Schläfe und das Hinterhaupt aufgesetzt. Sollte die Flickerantwort ausschliesslich von der optischen Hirnrinde herrühren, so müsste sie bei der letzteren Ableitung viel sicherer nachweisbar sein, dagegen bei der ersteren deutlicher auftreten, sollte der Netzhautprozess die uberwiegendere Rolle spielen. Wie in Abb. $10 \mathrm{~B}_{1}$ und $\mathrm{B}_{2}$ wiedergegeben ist, macht sich die Flickerantwort bei der NasenwurzelSchläfe-Ableitung sehr ausgeprägt bemerkbar, wenn die Frequenz etwa 10 pro Sek. nicht überschreitet. Einzelne Ausschläge sind bei verhältnismässig niederer Frequenz mit der typischen Form des Elektroretinogramms versehen, welche aber mit zunehmender Flickerfrequenz undeutlicher wird. Auch die Amplitude einzelner Ausschläge wird mit der Zunahme der Flickerfrequenz stark reduziert. 


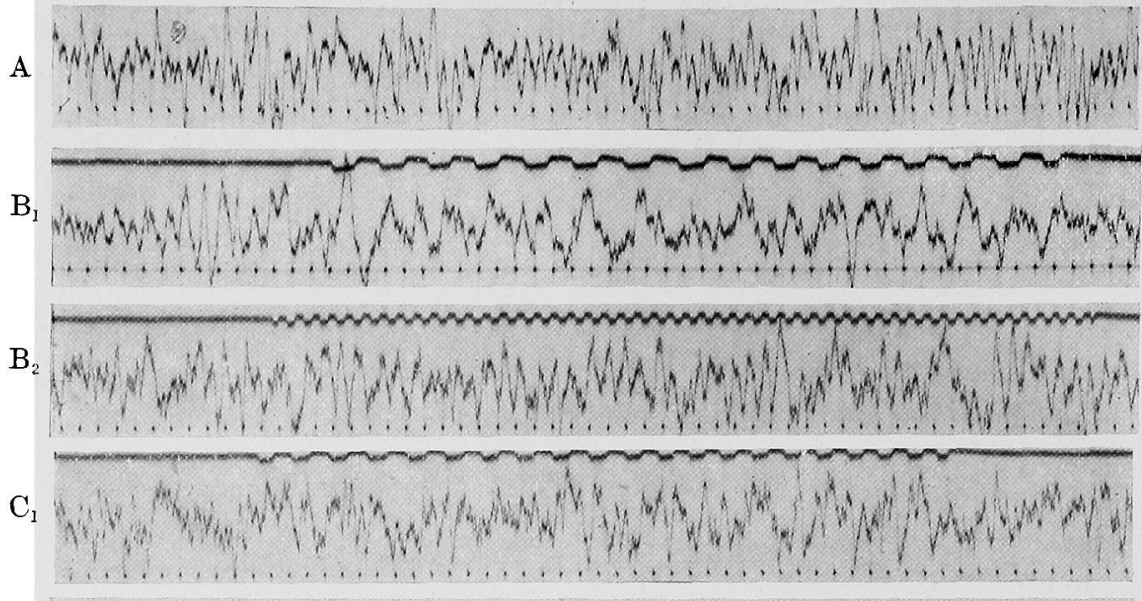

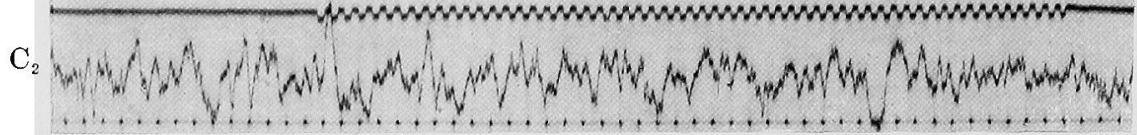

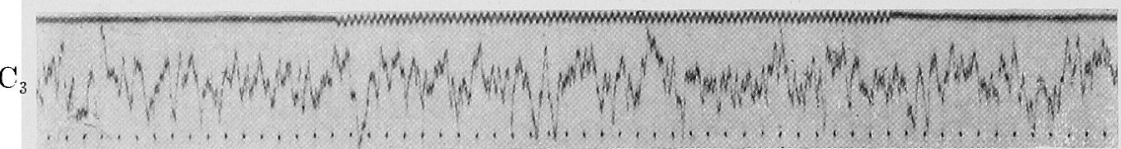

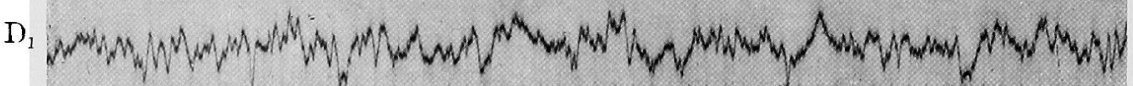

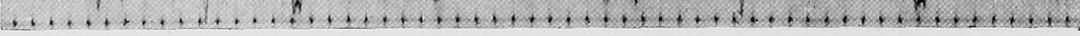
-

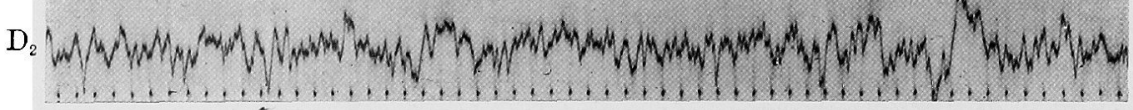

Abb. 10. Ergebnisse bei rhythmischer Augenbelichtung.

Die Belichtungsphase ist durch nach unten gerichtete Verschiebung des Signals aus einer Photozelle angezeigt (oberste Linie). Zeit in 1/5 Sek.

A : Kontrollkurve im Dunkeln ohne Fixierpunkt. Ableitung, Stirn-Hinterhaupt. B : Ableitung, Nasenwurzel-Schläfe. C: Ableitung, Schläfe-Hinterhaupt. D : Bipolare Ableitung vom Hinterhaupt mit einer Elektrode auf Protub. occip. und mit einer anderen $5 \mathrm{~cm}$ oberhalb derselben. Ausgenommen von $\mathrm{A}$ wurde ein schwach leuchtender Fixierpunkt angewandt, so dass die normalen $\alpha$-Wellen nicht so stark wie bei A auftraten.

Was etwas Eigentümliches für die Flickerantwort der Netzhaut ist, kann man im Verhalten des ersten Ausschlags erblicken; er verhält sich ganz gleich wie der „On"-Effekt auf einen Einzelreiz oder Elektroretinogramm, und seine Amplitude bleibt fast unabhängig von 
der Flickerfrequenz konstant. Es ist noch hervorzuheben, dass an diesen ersten Ausschlag sich ein verhältnismässig langes Refraktärstadium auschliesst.

Die Flickerantwort scheint andererseits auch von der Belichtung und vom Adaptationszustand der Augen abhängig zu sein, so dass die vorliegende Untersuchung erst nach einer Stunde des Wartens im Dunkeln ausgeführt wurde. Als Lichtquelle wurde die 30-Watt-Lampe von Mazda benutzt, welche in einem von einer runden Mattscheibe (Durchmesser $5 \mathrm{~cm}$ und Durchlässigkeit etwa 0,6) versehenen Kasten eingeschlossen war. Der Abstand der Mattscheibe von den Augen betrug etwa $80 \mathrm{~cm}$, und ein das Flicker erzeugender Sektor wurde dazwischen angebracht. Bei den dunkeladaptierten Augen ist ein zu starkes Licht wie z. B. das der 100 Watt-Lampe nicht so wirksam wie ein schwächeres Licht, um eine deutliche Flickerantwort herbeizuführen, was vielleicht auf das so verursachte zu starke Refraktärstadium zurïckzuführen ist.

Das Flicker bei der Schläfe-Hinterhaupt-Ableitung ist nicht weniger deutlich ( $\mathrm{Abb} .10 \mathrm{C}_{1}, \mathrm{C}_{2}$ und $\mathrm{C}_{3}$ ) und in manchen Hinsichten von demjenigen bei der Nasenwurzel-Schläfe-Ableitung verschieden. Die Hauptunterschiede sind folgenden: 1. der typische erste Ausschlag bleibt ganz aus, 2. die Amplitude einzelner Ausschläge nimmt mit steigender Flickerfrequenz nicht so deutlich ab, sondern erscheint unter Umständen desto mehr zuzunehmen, je näher die Flickerfrequenz der Eigenryhthmik der $\alpha$-Wellen gleich kommt, was der sogenannten Synchronisierung von Adrian entsprechen mag; 3. die Verschmelzungsfrequenz scheint hierbei etwas höher als bei der Nasenwurzel-Schläfe-Ableitung zu sein. In dieser Weise steht dieser Befund dem bisher mitgeteilten Flickerbild sehr nahe, und es ist recht wahrscheinlich, dass es sich hierbei um einen Himprozess handelt. Sollte der Prozess an der Netzhaut eine überwiegende Rolle spielen, so müsste der Flickereffekt bei dieser Ableitung viel schwächer ausfallen, weil die beiden Elektroden hierbei hinter den Augen liegen. Das Ausbleiben des ersten typischen Ausschlags spricht entschieden für diesen Schluss.

Auch die Tatsache, dass sich die Flickerantwort bei der bipolaren Ableitung vom Hinterhaupt nachweisen lässt, gestattet uns ohne weiteres ihre retinogene Herkunft auszuschliessen (Abb. $10 \mathrm{D}_{1}$ und $\mathrm{D}_{2}$ ). Die Verschmelzungsfrequenz wurde bei unseren Untersuchungen nicht genau bestimmt, da es hierbei hauptsächlich auf die Entscheidung des Entstehungsorts der Flickerantwort ankam. Auf je- 
den Fall hat sich bei diesen Untersuchungen herausgestellt, dass der Entstehungsort der Flickerantwort nicht nur aus der Verteilung des Potentials auf der Oberfläche des Kopfes, sondern auch aus der Eigenschaft des Flickerbildes ausfindig gemacht wird.

Bedeutet die Flickeranwort eine Reihe der nach einander auftretenden „On"-Effekte, so kann man sagen, dass der "On"-Effekt von der Oberfäche des Kopfes ableitbar ist, aber der "On"-Effekt in diesem Sinne ist offenbar von dem in Frage stehenden verschieden, worauf wir in der Diskussion wieder zurückkommen werden.

\section{Diskussion der Ergebnisse.}

In den Arbeiten von Korn $\mathrm{m} \ddot{\mathrm{ulle}} \mathrm{r}^{1)}$ und $\mathrm{F}$ is che $\mathrm{r}^{2)}$ wurde eine Elektrode auf die Kornea eines Auges und die andere auf die kontralaterale occipitale Hirnrinde aufgesetzt. Wenn auch nichț angegeben ist, warum solch eine Ableitungsweise gewählt wurde, muss sie wenigstens für den Nachweis des „On"-Effektes bequem gewesen sein. Aber bei dieser Elektrodenanordnung macht sich der Belichtungsstrom der Netzhaut sicherlich geltend, wie oben erörtert. Obgleich diese Arbeiten in dieser Weise nicht einwandfrei sind, dürfte man die beobachteten Potentialänderungen nicht ganz auf den Belichtungsstrom der Netzhaut zurückführen. Denn nach Wang lassen sich derartige Potentialänderungen mittels der konzentrischen Nadelelektroden nach A drian und Bronk direkt von der Grosshirnrinde ableiten, wobei der Belichtungsstrom der Netzhaut wegen der lokalen Ableitung offenbar kaum in Betracht kommt.

Es handelt sich also darum, welcher der beiden Prozesse an der Netzhaut und an der Grosshirnrinde eine überwiegende Rolle spielt. Dies Verhältnis soll bei der Ableitung von der Kopfoberfläche umso mehr in Erwägung gezogen werden, als die Potentialänderung der Grosshirnrinde hierbei viel schwächer in den Messungskreis aufgenommen wird, als bei der direkten Ableitung von der Hirnoberfläche, so dass der Belichtungsstrom der Netzhaut umso deutlicher in den Vordergrund treten kann.

In den Arbeiten von Jasper ${ }^{6)}$ und Cruikshank $k^{7}$ liess sich der sogenannte ,On "-Effekt zwar bei der Hinterhaupt-Ohrlappen-Ableitung deutlich erkennen, aber nur eine Andeutung desselben machte sich bei der bipolaren Ableitung vom Hinterhaupt bemerkbar. Diese Tatsache legt nahe, dass der sogenannte „On"-Effekt von der Netzhaut, aber nicht von der optischen Hirnrinde herrührt.

Der Belichtungsstrom der Netzhaut kann insofern von den Pro- 
zessen des Sehzentrums nicht unabhängig sein, als nervöse Impulse von der Netzhaut nach dem Sehzentrum hin fortgeleitet werden; nach Bartle ${ }^{5)}$ ist ihr Zusammenhang wenigstens beim Kaninchen so innig, dass der Gipfel der Eintrittsschwankung vom Elektroretinogramm mit demjenigen des echten O.E. am Sehzentrum zeitlich zusammenfällt. Dies Verhältnis macht es fast unmöglich, die beiden Prozesse nur ihrer Frscheinungsform nach von einander zu unterscheiden, falls sie zusammengefügt mit registriert werden, wie es bei der Ableitung von der Kopfoberfläche der Fall sein kann. Bei Tierexperimenten lassen sich nicht nur der „On "-Effekt sondern auch der dementsprechende "Off"-Effekt bei Belichtung der Augen nachweisen (Bartley und Wang), und zwar tritt der letztere beinahe konstant und sogar deutlicher als der erstere auf (Bartley). Der dementsprechende Befund ist niemals beim Menschen gefunden worden, was einen auffallenden Unterschied zwischen den Ergebnissen bei Menschen und Tieren ausmacht.

Im Gegensatz dazu wird der "Off"-Effekt beim Elektroretinogramm des Menschen bei verhältnismässig schwachér oder kurzdauernder Belichtung ganz vermisst, und wenn ein solcher auch bei Belichtung von genügender Stärke und Dauer auftritt, ist er immer viel schwächer als der dementsprechende „On"-Effekt. Mit diesen Eigenschaften des Elektroretimogramm stimmt gerade das Verhalten des sogenannten „On"-Effektes (,evoked potential") beim Menschen überein. Wäre der sogenannte „On"-Effekt oder dergleichen wirklich der Aktionsstrom der optischen Hirnrinde, so müsste die sehr unwahrscheinliche Annahme gemacht werden, dass das Verhalten des menschlichen Aktionsstroms von demjenigen des tierischen ganz verschieden sei. Dürfte der sogenannte „O" der Netzhaut betrachtet werden, so würde dieser Widerspruch ohne weiteres fortfallen.

Der O.E. des Menschen steht andererseits auch mit den spontanen Schwankungen der Hirnströme (Feldeigenströme nach Kornmüller) in einem Zusammenhang; nach Mita ${ }^{8)}$ fällt der erste elektronegative Ausschlag des „On"-Effektes mit dem Beginn der Unterdrückung der $\alpha$-Wellen zeitlich beinahe zusammen, welche während des darauf folgenden positiven Ausschlags ganz unterdrückt bleiben, und erst nach seinem Ablauf wieder erscheinen (Jasper). Dieser Autor hat zur Erklärung dieser Erscheinung die von Gasser ${ }^{25)}$ gefundene Tatsache herangezogen, dass die durch Verletzung veranlassten spontanen Erregungen des peripheren Nerven während des posi- 
tiven Nachpotentials dauernd unterdrückt bleiben, und die positive Schwankung des "On"-Effektes in analoger Weise als eine Phase der herabgesetzten Erregbarkeit der nervösen Elemente am Sehzentrum betrachtet. Diese Analogie dürfte zwar solange bestehen, als sich der O.E. und die $\alpha$-Wellen auf ein und demselben Substrat abspielen sollten, aber davon könnte doch keine Rede sein, wären die beiden von ganz verschiedenem Ursprung.

Wie oben erwähnt, führt der O.E. zum vollständigen Verschwinden der $\alpha$-Wellen, hingegen können die letzteren auf den ersteren keinen Einfluss ausüben; z.B. tritt der „On"-Effekt dávon unabhängig konstant auf, ob die $\alpha$-Wellen voll entwickelt vorhanden sind, oder infolge irgend einer Ursache schon verlorengegangen sind, wie Cruikshankschon hinwies. Diese gerichtete Einwirkung ist fast unbegreiflich, setzt man voraus, dass die beiden Prozesse bei ein und demselben nervösen Elemente der optischen Hirnrinde vor sich gehen, wie Jasper behauptet. Im Gegensatz dazu ist sie leicht verständlich, wenn man sich den Sitz des „On"-Effektes etwas in die Peripherie gegen den der $\alpha$-Wellen verlegt denkt, denn die Impulse sollen bei der Sinneserregung immer von der Peripherie nach dem Zentrum, aber nicht umgekehrt fortgeleitet werden.

Auf jeden Fall ist der sogenannte „On"-Effekt als ein zentraler Prozess sehr schwer zu verstehen, aber wir behaupten weder, dass der "On"-Effekt der optischen Hirnrinde beim Menschen überhaupt nicht vorhanden ist, noch dass er beim uneröffneten Schädel keineswegs nachweisbar sein wird. Vielmehr sind wir der Ansicht, dass er nach einer geeigneten Methode von der Oberfläche der Kopfhaut ableitbar ist, wofür der oben ausgeführte Flickerversuch einigermassen spricht.

Wir haben auch als ein wertvolles Nebenprodukt dieser Untersuchung eine neue Ableitungsmethode des Elektroretinogramms des Menschen kennengelernt. Bei bisherigen Versuchen wurde die differente Elektrode immer auf die Oberfläche der Kornea aufgesetzt, und dabei erhielt man das Belichtungspotential als eine Schwankung von etwa einigen hundert $\mu \mathrm{V}$. Aber das Aufsetzen der Elektrode auf die Kornea war für die Versuchsperson so quälend, dass sie langdauernde oder wiederholte Untersuchungen kaum ertragen konnte. Ein anderer Übelstand war, dass die Elektrode schon bei geringen Bewegungen der Augen leicht rutschte und so schwere Störungen hervorrief.

Bei unserer Methode wird die differente Elektrode auf die unbewegliche Stelle der Gesichtshaut (Nasenwurzel) fixiert, so dass die 
Elektrode nicht nur der Versuchsperson keine Qual macht, sondern auch bei Augenbewegungen keine Verschiebung erfährt. Man vermag also stundenlang ebenso bequem wie bei der Elektrenkephalographie die Untersnchung fortzusetzen, was von der bisherigen Methode nicht gesagt werden kann. Aber auch die unsrige ist nicht ohne Nachteile: Infolge der Ableitung von der Hautoberfläche wird die EMK ziemlich stark reduziert, und andererseits ist die Beimengung der Hirnströme schwer zu vermeiden.

\section{Zusammenfassung.}

Bei der Elektrenkephalographie des Menschen lässt sich eine von den eigentlichen Hirnströmen sicher unterscheidbare langsame Schwankung auf Augenbelichtung hin nachweisen. Da sie dem bei Tieren gefundenen "On"-Effekt der optischen Hirnrinde etwas ähnlich ist, wurde sie ohne weiteres der Grosshirnrinde zugeschrieben. Angesichts ihres mit dem cerebralen „On"-Effekt bei Tieren nicht ganz übereinstimmenden Verhaltens wurde ihre vermeintliche Herkunft in Zweifel gezogen und folgende Untersuchung angestellt, um das Wesen dieser Potentialschwankung aufzuklären :

1. Sie ist von Lid- und Augenbewegungen unabhängig, was mit der elektrischen Registriermethode der Lid- und Augenbewegungen einwandfrei nachgewiesen wurde.

2. Ein rotes Licht ist weniger wirksam als ein weisses oder ein blaues, um diese Schwankung hervorzurufen.

3. Durch Untersuchungen über die Potentialverteilung auf dem Kopf wurde festgestellt, dass diese Schwankung von den Augen oder wenigstens von ihrer Nähe herrührt.

4. Der naturgetreue Verlauf dieser Schwankung wurde mittels eines Gleichstromverstärkers und eines Oszillographen registriert, um festzustellen, dass sie in jeder Hinsicht mit dem Elektroretinogramm des Menschen übereinstimmt.

5. Auf rhythmische optische Reize lassen sich in Bestätigung des Adrian schen Befunds periodische Schwankungen des Potentials nachweisen. Das Bild und andere Eigenschaften dieser Flickerantwort sind auf der vorderen und der hinteren Hälfte des Kopfes sehr verschieden, indem Netzhautprozess beim ersteren Fall eine überwiegende Rolle spielt.

Es sei nachdrücklich betont, dass sich der Belichtungsstrom der Netzhaut bei der Elektrenkephalographie unter Umständen geltend 
macht, um eine Konfusion mit Hirnströmen hervorzurufen, besonders wenn eine Elektrode in der Nähe der Augen liegt.

Dem Unterrichtsministerium sind wir für das Zustandekommen dieser Arbeit durch seine finanzielle Unterstützung zu herzlichem Dank verp flichtet.

\section{Literatur.}

(1) Kornmüller, A. E., J. Psyehol. u. Neurol., 1932, 44, 447.

(2) Fis che r, H. M., Pflugers Arch., 1932, 230, 161.

(3) Bartle y, S. H. und G. H. B is h o p, Am. J. Physiol., 1933, 103, 159.

(4.) Wang, G. H., Chinese J. Physiol, 1934, 8, 121.

(5) Bartley, S. H., Am. J. Physiol., 1936, 117, 338.

(6) Jasper, H. H., Simposia on quantitative biology, Cold Spring Harbor, L. I., N. Y., 1936, 4, 320.

(7) Cruikshank, R. M., J. Exp. Psjchol, 1937, 21, 625.

(8) Mita, T., Tohoku J. Exp. Med., 1941, 39, 485.

(9) Rohracher, H., Commentationes Pontificia Acad. Sci., 1938, 2, 225. Zitiert aus H. D a vis, Ann. Review of Physiol., 1939, 1, 354.

(10) Sch ott, E., Deutseh. Arch. f. klin. Med., 1922, 140, 79.

(11) Myers, I. L., Arch. Neurol. a. Psychiat,, 1929, 21, 901.

(12) Mowrer, O. H., T. C. Ruch u. N. E. Miller, Am. J. Physiol., 1936, 114, 423.

(13) Fenn, W. O. u. J. B. Hurs h, Am. J. Physiol., 1937, 118, 8.

(14) Adrian, E. D. a. B. H. C. Matthews, Brain, 1934, 57, 355.

(15) Himstedt, F. u. W. A. Nagel, Ber. Naturf. Ges. Freiburg i. Br., 1901, $11,153$.

(16) Piper, H., Arch. Physiol, 1904, 470.

(17) Brossa, A. u. A. Kohlrausch, Arch. Physiol., 1913, 449.

(18) Fröhlich, F. W., Zeits. Sinnesphysiol., 1913, 48, 147.

(19) S achs, E., Handb. d. norm. u. path. Physiol. XII/2, S. 1464, Berl. 1931.

(20) Hartline, H. K., Am. J. Physiol, 1925, 73, 600.

(21) Gröppel, F., F. Ha ass u. A. Kohlrauseh, Zeits. Sinnesphysiol., 1938, 67, 207.

(22) Loomis, A. L., E. N. Harvey n. G. Hobart, J. Exp. Psychol., 1936, 19, 249.

(23) Rohracher, H., Zeits. Psychol., 1937, 140, 274.

(24) Kornmüller, A. E., Zeits. Sinnesphysiol., 1940, 68, 119.

(25) Gasser, H. S., J. Physiol., 1935, 85, 15 P. 